\title{
Alternating Coordinate-Momentum Representation for Quantum States Based on Bopp Operators for Modelling Long-Distance Coherence Aspects
}

\author{
Ezzat G. Bakhoum ${ }^{1}$ and Cristian Toma ${ }^{2}$ \\ ${ }^{1}$ Department of Electrical and Computer Engineering, University of West Florida, 11000 University Parkway, Pensacola, FL 32514, USA \\ ${ }^{2}$ Faculty of Applied Sciences, Politehnica University, 315 Splaiul Independentei, 060042 Bucharest, Romania
}

Correspondence should be addressed to Cristian Toma; cgtoma@physics.pub.ro

Received 6 August 2014; Accepted 22 August 2014

Academic Editor: Florin Pop

Copyright (C) 2015 E. G. Bakhoum and C. Toma. This is an open access article distributed under the Creative Commons Attribution License, which permits unrestricted use, distribution, and reproduction in any medium, provided the original work is properly cited.

\begin{abstract}
This study presents an alternating coordinate-momentum representation for propagation and transition of associated wave function, based on Bopp operators and on a certain symbolic determinant corresponding to a set of two linear equations with null free terms. It is shown that this alternating representation can justify in a good manner the patterns created through reflection/refraction of waves on nonperfectly smooth interfaces and phase correspondence of diffracted beams without the need of supplementary support functions. Correlations with Lorentz transformation of wave functions by interaction with a certain material medium (the space-time origin of a wave-train being adjusted) are also presented, and supplementary aspects regarding the use of electromagnetic scalar and vector potentials for modelling evolution within this alternating representation are added.
\end{abstract}

\section{Introduction}

As it was shown in [1], the study of light wave propagation phenomena at the interface between two different media (reflection/refraction) based on wavefronts generated by multiple centers of reflection/refraction situated on this interface requires an explanation regarding further propagation along directions normal to these wavefronts. For an oblique incidence of a plane wave, a certain center of reflection/refraction will be the first one which emits wavelets with the of speed of light specific to that material medium. Until it interacts with the wavefront generated by another center of reflection/refraction, we should consider that parts of the incident wave are radiated along all spatial directions. The procedure can continue by analyzing the interaction of each newly created wavefront with subsequent wavelets, implying that each time a supplementary part of the received wave will be transmitted on spatial directions which differ to the main axis of reflection/refraction.

Moreover, the assumption regarding the constant phase $\operatorname{shift}(\pi$ for electric field $\mathbf{E}$, e.g.) for reflected/refracted wave in any surface point is also questionable, since the interface is far from being perfectly smooth and perfectly conductive. A certain transient time interval for creating the electrostatic equilibrium is always required, so as the electric field $\mathbf{E}$ to vanish on this surface. As a consequence, local phase shifts for reflected/refracted wave can not be avoided within this local mathematical model. According to the linear wave equation (with constant coefficients), these could generate multiple inphase local waves propagating along spatial directions which differ for the main reflection/refraction axis. Thus parts of reflected/refracted wave would be transmitted in a large solid angle and directionality would be lost within a very short length interval.

According to solid state theory, a very good argument regarding the perfectly smooth approximation for reflection/refraction theory and for the constant phase shift consists in the fact that photons usually interact with collectivised electrons of the solid crystalline lattice before being reemitted. These electrons could be considered as moving tangent to the interface since sudden changes of trajectory could generate significant electromagnetic field (accelerations being involved). This picture is supported also by quantum physics, since the associated-wave function for 
the collectivised electrons is represented in position for large space intervals, the influence of local nonuniformities being decreased. Thus can be drawn a tangent line with a great radius of curvature, resulting in a better directionality for reflected/refracted beams and the same phase shift for them.

However, this possible explanation does not take into account the constant phase shift between the incident and the reemitted beams from points situated far apart from each other, when the associated-wave function for collectivised electrons can not be considered as being the same anymore. There is no perfect crystal lattice, so the quantum functions corresponding to collectivised electrons or to vibrations (phonons) are spatially limited. Moreover, when supplementary diffraction phenomena are involved; some points becoming secondary wave-sources (reemitting light beams) are situated on nonadjacent surfaces (see the edges of a diffraction grating) and they can not be correlated by any surface quantum wave functions.

In [1], an internal report published later in scientific journals in 2013 suggested that a kind of support waves is generated on this interface. They act upon reflecting/refracting points and correlate the phase of reemitted waves by interface points situated at great distance. These support waves could also correlate nonadjacent space intervals wherefrom secondary light beams are emitted through diffraction. So as to be effective, this possible intuitive and computational model requires a high speed for these propagating support waves in order to regroup the wavefronts into light beams with certain directionality within an extremely short time interval. The use of momentum space representation for the light wave is also recommended for describing phase correlation and coherence phenomena.

The wave-vector (momentum) values generated through these support functions represent the base for wave propagation on next time intervals. Based on wave equation

$$
\frac{\partial^{2} \phi}{\partial x^{2}}+\frac{\partial^{2} \phi}{\partial y^{2}}+\frac{\partial^{2} \phi}{\partial z^{2}}=\frac{1}{v^{2}} \frac{\partial^{2} \phi}{\partial t^{2}}
$$

a set of possible high speed propagating functions as

$$
\phi(x, y, z, t)=a x+b y+c x+d t+e,
$$

where quantities $a, b, c, d, e$ are constant values, were proposed as support functions. This corresponds with a timedependent plane equation, a plane which moves in the threedimensional space. The value of $\phi$ at space-time origin (when $x=y=z=t=0$ ) can be encountered for $t>0$ on a set of planes situated at distance

$$
D=\left|\frac{0 a+0 b+0 c+(d t)}{\sqrt{a^{2}+b^{2}+c^{2}}}\right|=\left|\frac{d t}{\sqrt{a^{2}+b^{2}+c^{2}}}\right|
$$

considered from space origin (according to basic analytical geometry). As a consequence, the plane on which $\phi$ equals $e$ (passing through origin at zero time moment) moves in space with velocity

$$
w=\frac{D}{t}=\left|\frac{d}{\sqrt{a^{2}+b^{2}+c^{2}}}\right| .
$$

There being no restriction regarding the choice for the set constant values $a, b, c$, and $d$ results in that (unlike standard solutions of wave equation) these plane equations can be represented as constant values propagating in space-time with any velocity (the speed $w$ is not correlated with the wave equation anymore).

However, the arbitrary choice of these constants is not consistent with basic laws and principles of both classical and quantum theory of light waves (being similar to the attempt of defining some hidden variables). An attempt for justifying different actions connected to coordinate and momentum representations by presenting coordinate and momentum operators as acting in different spaces (reciprocal Fourier spaces) has been first performed in [2], with the requirement of considering light speed $c$ as limit speed for transmitting interaction at quantum level, for variables corresponding with the same quantum wave function (later this attempt was defined as the Sterian realistic approach, being connected to Schwinger principle in extended studies published in 2013). Yet this implies the use of a supplementary inner wave for transmitting information inside a certain wave (the light wave which is analysed). This is the same as using hidden variables (in this case hidden waves).

However, in [3] (an internal report published later in 2013) it was shown that uncertainty principle in quantum physics allows a kind of instantaneous propagation of interaction. The analysis is based on creation/annihilation phenomena. The annihilation of the particle implies the instant annihilation of all parts of this wave-train, irrespective to the distance to this interaction point. It is true that this seems to contradict the relativity postulates (no speed can surpass the light speed) but in fact any speed can be noticed just by an emmission and a reception of a certain signal or particle (not just by a single emission or a single annihilation). Since the particle ceases to exist, its final momentum is zero. There being no uncertainty, it results that

$$
\Delta p_{x}=\Delta p_{y}=\Delta p_{z}=0
$$

and, according to uncertainty principle,

$$
\begin{aligned}
& \Delta x \geq \frac{\hbar}{\Delta p_{x}} \longrightarrow \infty, \\
& \Delta y \geq \frac{\hbar}{\Delta p_{y}} \longrightarrow \infty, \\
& \Delta z \geq \frac{\hbar}{\Delta p_{z}} \longrightarrow \infty .
\end{aligned}
$$

Thus this kind of annihilation phenomenon could be considered as being infinitely extended. The uncertainty for momentum determines the uncertainty for space intervals. However, in [3] it was also shown that this aspect can not be automatically extended for transmitting fast information necessary for phase correlation or possible regrouping of light beams. Usually deflections (including any possible reflections/refractions or diffractions) are considered to alter just the wave-vector (the momentum) for parts of the spatially extended wave function, without any change regarding the 
coherence (phase) of these beams. These phenomena can not be put in correspondence with any creation/annihilation of particles (despite the fact that the wave-vector is altered for parts of the associated wave); otherwise any reflection/refraction or diffraction of a part of the incident wave would generate an annihilation of the entire associated wavefunction in any other point of space.

As a consequence, an improved intutive and computational model able to include quantum aspects generated by uncertainty principle regarding a high speed for transmitting interaction and spatial-temporal coherence achieved for deflected parts of the same wave function still has to be determined. For this purpose, this study will show that a certain alternating representation for the wave function can justify in a good manner the patterns created through reflection/refraction of waves on nonperfectly smooth interfaces and phase correspondence of diffracted beams without the need of supplementary support functions. Moreover, this alternating coordinate-momentum representation is suggested by Lorentz transformation of wave trains within special relativity, since the space-time origin for this transformation can not be considered as corresponding to the first interaction with the observer's material medium (as presented in [4]). A very low value for the received signal at zero moment could easily correspond to a noise from the environment, so parts of the received wave-train around this moment could be considered to belong to different wavefunctions, not being transformed as a whole (the synchronization moment would differ).

The problem of a superposition of different light (electromagnetic) signals is also connected to the case when a charged particle (an electron for example) moves in an electrostatic field. According to auantum mechanics, within Schrodinger's equation some supplementary terms should be added for momentum $\vec{p}$ and energy $E$ as

$$
\begin{aligned}
& \vec{p} \longrightarrow \vec{p}-q \vec{A}, \\
& E \longrightarrow E-q V
\end{aligned}
$$

in scaled measurement units (the light speed $c$ at the denominator of second term in previous equation was set to unity for simplicity).

However, if aspects from quantum field theory are added (second quantization implying creation/annihilation of particles), then problems regarding a phase-loss phenomena are generated. Any local computed probability of interaction with virtual photons (resulted from electrostatic field decomposition) requires a previous transient time (so as physical quantities as wavelength and angular fequency to be defined on a certain time interval) and thus the timelength of the wave-train associated with the final electron tends to zero, a vanishing phenomenon for the initial electron in contradiction with experimental facts. As a consequence, the electromagnetic field (characterized by electric and magnetic fields $\vec{E}$ and $\vec{B}$ or by scalar and vector potentials $V$ and $\vec{A}$ ) should be preliminary determined in a certain area before the suggested alternating coordinate-momentum representation to be taken into consideration.

\section{Long-Distance Coherence Phenomena Based on Bopp Operators}

Starting from standard coordinate and momentum representations in quantum mechanics, it was shown that there is not a unique way to represent a quantum state as a wave function $\Psi=\Psi(\Gamma)$, where $\Gamma$ represents a point in the classical phase space $q, p$ (position and momentum). Using an integral transformation as

$$
\Psi(\Gamma)=\int K\left(\Gamma ; q^{\prime}\right) \Psi\left(q^{\prime}\right) d q^{\prime}
$$

where $K\left(\Gamma ; q^{\prime}\right)$ corresponds to an integral kernel, another representation can be obtained from the standard coordinate representation $\Psi(q)$. An example is the kernel

$$
K_{\mathrm{CS}}=\left(\lambda^{2} \pi\right)^{-1 / 4} \exp \left(-\frac{\left(q-q^{\prime}\right)^{2}}{2 \lambda^{2}}-\frac{i p}{\hbar}\left(q^{\prime}-q\right)\right)
$$

( $\lambda$ being the natural length scale defined by the mass and frequency of the oscillator) able to generate the Glauber coherent states (eigenstates of the annihilation operator). The Gaussian factor is required by normalization. This kernel $K_{\mathrm{CS}}$ was used for constructing phase space representation of quantum mechanics of Torres-Vega and Frederick [5, 6]. They have shown that if $\Psi(q, t)$ satisfies the standard Schrodinger equation

$$
i \hbar \frac{\partial}{\partial t} \Psi(q, t)=\left[-\frac{\hbar^{2}}{2 m} \frac{\partial^{2}}{\partial q^{2}}+U(q)\right] \Psi(q, t)
$$

then $\Psi(\Gamma)$ obtained through the integral transformation based on $K_{\mathrm{CS}}$ satisfies a kind of Scrodinger's equation in phase space as

$$
i \hbar \frac{\partial}{\partial t} \Psi(\Gamma, t)=\left[-\frac{\hbar^{2}}{2 m} \frac{\partial^{2}}{\partial q^{2}}+U\left(q+i \hbar \frac{\partial}{\partial p}\right)\right] \Psi(\Gamma, t) .
$$

Moreover, if a gauge transformation as

$$
\Psi^{\prime}(q, p)=\exp \left(\frac{-i p q}{2 \hbar}\right) \Psi(q, p)
$$

is performed, the symmetric formula

$$
i \hbar \frac{\partial}{\partial t} \Psi^{\prime}(\Gamma, t)=\left[\frac{1}{2 m} \widehat{P}^{2}+U(\widehat{Q})\right] \Psi^{\prime}(\Gamma, t),
$$

where

$$
\begin{aligned}
& \widehat{Q}=\frac{q}{2}+i \hbar \frac{\partial}{\partial p}, \\
& \widehat{P}=\frac{p}{2}-i \hbar \frac{\partial}{\partial q}
\end{aligned}
$$

satisfying $[\widehat{Q}, \widehat{P}]=i \hbar$ according to canonical commutation relations. 
Being convenient to scale phase space variables $(q, p) \rightarrow$ $(2 q, 2 p)$, it results in

$$
\begin{aligned}
& \widehat{Q}=q+\frac{i \hbar}{2} \frac{\partial}{\partial p}, \\
& \widehat{P}=p-\frac{i \hbar}{2} \frac{\partial}{\partial q} .
\end{aligned}
$$

These are the so-called Bopp operators. Their utility is connected to quantum jumps, due to the additional term added to standard quantum operators $q$ and $p$. However, we must point the fact that these Bopp operators are very useful for presenting fast long-range coherence phenomena suggested by operators $q$ (position) and $p$ (momentum) in quantum phyiscs without any creation/annihilation phenomena to be involved (as considered in [3]). For space areas with the same energy (connected to frequency) and/or momentum (connected to wave-vector) it results in the possiblity of fast long-range phenomena for transmitting information for parts of the same wave function.

This aspect can be proved by the action of operator $\widehat{Q}$ upon a certain function $\phi$ with the same wave vector in an extended spatial area. According to previous relations, it results in

$$
d p \longrightarrow 0 \Longrightarrow \frac{\partial}{\partial p} \phi \longrightarrow \infty \Longrightarrow \widehat{Q} \phi \longrightarrow \infty .
$$

Thus the action of operator $\widehat{Q}$ (used instead of coordinate operator $q$ in standard quantum relations) is extended to long-range distances (theoretically to infinite) within the equations for the same wave function (possibly extended to nonadjacent space areas due to deflecting phenomena previously mentioned). This allows fast-speed correlations for parts of the same wave function.

For example, the wave front of a certain monochromatic planar light beam propagating along $x_{i}$ axis presents a null variation of the wave vector along the $x_{j}$ axis normal to $x_{i}$. This corresponds to the same wave vector $k_{j}$, implying the same momentum $p_{j}$ along $j$ axis also. It results in $\Delta p_{j}=$ 0 , which means that $\widehat{Q}_{j} \rightarrow \infty$ when a change in the quantum function $\Psi$ occurs along $i$ axis due to a possible reflection/deflection (in the limit case, a nonzero difference $\Delta \Psi$ is divided by an approximately zero difference $\Delta p_{j}=0$ ). Thus a fast long-range interaction can be noticed between all points situated on the planar wave front when a sudden change occurs for one of them, the long-distance phase correlations being justified. For the case of electromagnetic interaction, these changes are connected to the influence of electromagnetic fields (usually represented by scalar potential $V$ and vector potential $\vec{A}$ ) in a certain area. This local action is long-distance transmitted by means of this Bopp operator $\widehat{Q}$.

In a similar manner, Bopp operators can be used for justifying regrouping aspects within the same wave packet corresponding to an associated wave-function. Within quantum physics, the motion of a particle is described as an almost monochromatic wave function, these means as a superposition of waves with angular frequency $\omega$ and wave vector $k$ situated within some limited intervals. The maximum amplitude for these sets of waves corresponds with a dominant component of angular frequency $\omega_{0}$ and wave vector $k_{0}$. The other components are considered to possess lower amplitudes symmetrically disposed as decreasing functions around these central values in this energy-momentum space.

The motion of this wave packet is described by the socalled group velocity, defined as

$$
v_{g}=\frac{d \omega}{d k}
$$

and (according to correspondence angular frequency-energy and wave vector-momentum) as

$$
v_{g}=\frac{d E}{d p} .
$$

The derivatives with respect to $k$ or $p$ are considered for the central values of angular frequency $\omega_{0} \leftrightarrow$ energy $E_{0}$ and wave vector $k_{0} \leftrightarrow$ momentum $p_{0}$.

Since the function amplitude versus momentum presents a maximum value for $p=p_{0}$, it results that $d p$ equals zero around this central value. As a consequence, the term $\partial \Psi / \partial p$ within Bopp operator $\widehat{Q}$ (corresponding to spatial coordinate q) tends to infinite as in the previous case, for any nonzero change of quantum wave function $\Psi$ (a quantum jump). A long-distance action of this dominant component upon the other lower amplitude components within this superposition can be considered, with possible effects upon regrouping aspects of quantum wave functions (spatial dispersion due to different velocities being not allowed).

This aspect is also consistent with Lorentz transformation of wave trains within special relativity, since the space-time origin for this transformation can not be considered as corresponding to the first interaction with the observer's material medium (as presented in [4]). It can be easily considered that a very low value for the received signal at an initial time moment represents a noise from the environment. Thus parts of the received wave-train around this initial moment could be considered to belong to different wave functions at the very beginning, not being transformed as a whole (the synchronization moment would differ). As a consequence, a further interaction between these already transformed low-amplitude parts and the central high-amplitude part of the same wave function is required, so as the spacetime coordinates to be transformed as a whole for the entire wave-function. The synchronization moment (the zero moment for the Lorentz transformation) should be the same for all these parts (considered along time axis) and it is most convenient to define this time origin as the moment when this high-amplitude central part (where momentum $p$ is almost constant and thus $\Delta p$ is approximately equal to zero on an extended time interval) interacts with the observer's material medium. An extended temporal interval with almost constant momentum $p$ could generate later regrouping phenomena based on the same Bopp operator $\widehat{Q}$ which tends to infinity when $\Delta p$ is almost zero for a wave function altered through interaction in a very short time interval. 
It is true that this aspect would have been suggested by the Heisenberg uncertainty principle also, according to

$$
\Delta q \Delta p \geq \frac{\hbar}{2}
$$

However, in the most general case this principle is connected to measurement aspects and does not imply inner dynamics for the quantum wave function. On the contrary, the use of these Bopp operators is related to certain inner dynamics of the wave function, aspects regarding the range of internal interaction corresponding to it being covered. Moreover, the operators generated by variables $q$ and $p$ are mixed together in an almost symmetrical form.

It can be noticed that $p$ and $q$ operators can be encountered both under: (i) multiplicative form-the operator written at the numerator of a multiplicative factor acting to the right/left upon a ket or bra state vector, and (ii) differential form-the operator written at the denominator of a differential expression acting to the right/left upon a ket or bra state vector (according to Dirac notations). Unlike multiplicative operators, the differential operators can not be considered as acting almost instantaneously in any intuitive or computational (discretized) model. A kind of memory for certain variables on previous time intervals is required. This aspect of any discretized computational model intending to solve a differential equation can be easily extended to quantum models based on Bopp operators, where discontinuities (quantum jumps) could be involved.

Moreover, the symmetrical form of Bopp operators as related to $q, p$ operators suggests that these coordinatemomentum operators $q$ and $p$ should be used at the same time in a quantum equation of evolution. Yet this could correspond to an increased number of variables/operators used in the same equation. So a method for analyzing equations based on Bopp operators is required. As will be shown in next paragraph, a possible choice is represented by the use of symbolic determinants for solving a set of linear equations for the case when the free terms tend to zero (the limit being an undetermined set of equations with possible nontrivial solutions). As the source of these quantum jumps, terms corresponding to external electromagnetic fields should be inserted.

\section{A Symbolic Determinant for Alternating Coordinate-Momentum Representation}

Let us consider that two real functions should satisfy (in the limit case) a homogenous linear system of equations with null free terms as

$$
\begin{gathered}
\widehat{a} \phi+\widehat{b} \eta=0, \\
-\widehat{b} \phi+\widehat{a} \eta=0,
\end{gathered}
$$

where $\widehat{a}$ and $\hat{b}$ are not constant coefficients, but linear operators. Trying to solve this system by reduction method, the first step consists in amplifying first equation by operator $\widehat{b}$ to the left and the second equation by operator $a$ also to the left. It results in

$$
\begin{gathered}
\widehat{b} \widehat{a} \phi+\widehat{b}^{2} \eta=0, \\
-\widehat{a} \widehat{b} \phi+\widehat{a}^{2} \eta=0 .
\end{gathered}
$$

Next step consists in performing the sum of these two equations. It results in

$$
[\widehat{b} \widehat{a}-\widehat{a} \widehat{b}] \phi+\left[\widehat{b}^{2}+\widehat{a}^{2}\right] \eta=0 .
$$

From now on, this equation will be referred to as Complete Equation. If $\widehat{a}$ and $\widehat{b}$ are commutative operators, then first term vanishes in this complete equation, so the second term will be

$$
\left[\widehat{b}^{2}+\widehat{a}^{2}\right] \eta=0
$$

This equation is similar to the wave equation written in the cuadridimensional space, where operators $\widehat{b}$ and $\widehat{a}$ correspond to partial differentiation with respect to a certain space coordinate ( $x$ for example) and to $i c t$, respectively ( $t$ representing time and $c$ corresponding to light speed in vacuum). It should be noticed that these differentiation operators satisfy the commutative relation $\widehat{b} \widehat{a}-\widehat{a} \widehat{b}=0$ and so the complete equation will correspond to a simple propagation equation.

However, if $\widehat{b}$ and $\widehat{a}$ are noncommutative operators, this means

$$
\widehat{b} \widehat{a}-\widehat{a} \widehat{b}=1
$$

in scaled measurement units; then the first term in complete equation previously presented does not vanish. Moreover, this first term in complete equation can be moved to righthand side (with opposite sign) and becomes a kind of source (a free term) for the second term, as

$$
\left[\widehat{b}^{2}+\widehat{a}^{2}\right] \eta=[\widehat{a} \widehat{b}-\widehat{b} \widehat{a}] \phi .
$$

The left-hand side operator acting upon function $\eta$ is the symbolic determinant $\widehat{S}$ for the linear set of equations.

A similar equation (with a similar left-hand side quadratic expression and a right-hand side nonzero value) can be encountered for the hamiltonian function of the quantum harmonic oscillator. For this case operators $\widehat{b}$ and $\widehat{a}$ correspond usually to a coordinate operator and to differentiation with respect to this coordinate (the quantum momentum operator), so this hamiltonian $\widehat{H}$ equals

$$
\widehat{H} \eta=\left(\frac{\partial^{2}}{\partial x^{2}}+x^{2}\right) \phi
$$

in scaled measurement units (the constants in front of partial differentiation operators and in front of coordinate operators were set to unity for simplicity).

At first sight this seems to be pure algebra or at least symbolic algebra for representing the action of operators in quantum mechanics. No inner dynamics for a wave function 
could be noticed. However, a decomposition of operators $\widehat{a}$ and $\widehat{b}$ according to

$$
\begin{aligned}
& \widehat{a}=\widehat{a}_{i}-\widehat{a}_{f}, \\
& \widehat{b}=\widehat{b}_{i}-\widehat{b}_{f}
\end{aligned}
$$

can be performed (the minus sign and the indices will be justified later). By substitution, it results in

$$
\begin{gathered}
\left(\widehat{a}_{i}-\widehat{a}_{f}\right) \phi+\left(\widehat{b}_{i}-\widehat{b}_{f}\right) \eta=0, \\
\left(-\widehat{b}_{i}+\widehat{b}_{f}\right) \phi+\left(\widehat{a}_{i}-\widehat{a}_{f}\right) \eta=0
\end{gathered}
$$

which can be written also as

$$
\begin{gathered}
\widehat{a}_{i} \phi+\widehat{b}_{i} \eta=\widehat{a}_{f} \phi+\widehat{b}_{f} \eta, \\
-\widehat{b}_{i} \phi+\widehat{a}_{i} \eta=-\widehat{b}_{f} \phi+\widehat{a}_{f} \eta .
\end{gathered}
$$

The right-hand side of these two previous equations (where index $f$ can be encountered for $\widehat{a}$ and $\widehat{b}$ operators) could be considered as being determined by the left-hand side of them. It looks like the operators $\widehat{a}_{i}$ and $\widehat{b}_{i}$ act upon a pair of functions $\phi$ and $\eta$ so as to determine the right-hand side of these two previous equations. Since this right-hand side depends upon the action of operators $\widehat{a}_{f}$ and $\widehat{b}_{f}$ upon the same functions $\phi$ and $\eta$ considered at a bit later time moment, the value of the two functions $\phi$ and $\eta$ at the later time can be further determined.

Certain inner dynamics of the wave function work so as to obtain a minimum difference between functions $\phi$ and $\eta$ at the initial time moment and the same functions $\phi$ and $\eta$ considered at this later time moment. If the time difference between the initial moment of time (considered in left-hand side) and the final moment of time (the later moment of time, considered in right-hand side) is extremely small (orders of magnitude less than the infinite small time intervals required by the use of differential operators as $\partial / \partial q$ or $\partial / \partial p$ for quantum states), it will be not necessary to use different notations or supplementary indices for functions $\phi$ and $\eta$ in left- and right-hand sides of these two previous equations (indices $i$ and $f$, with the meaning of initial and final, respectively, being kept just for operator decomposition).

By moving right-hand side terms to the left in both previous equations the set of linear equations with null free term presented at the beginning of this paragraph will be obtained. This shows that the symbolic determinant similar to cuadridimensional propagation equation (the second part of the complete equation) and the commutative or noncommutative rules (the first part of the complete equation) could be generated by an inner dynamics of quantum functions, a quick path from some initial functions $\phi_{i}, \eta_{i}$ to certain final functions $\phi_{f}, \eta_{f}$ being involved.

For determining the operators $\widehat{a}_{i}, \widehat{a}_{f}$ and $\widehat{b}_{i}, \widehat{b}_{f}$ to be placed hand and right-hand side on the previous set of equations the standard decomposition of hamiltonian operator in a sum of the kinetic energy operator (usually a differential operator) and the potential energy operator (usually a multiplicative operator) could be used. This suggests the use of operators as

$$
\begin{gathered}
\widehat{a}_{i}=\frac{\partial}{\partial x}, \quad \widehat{b}_{i}=\frac{\partial}{\partial(i c t)}, \\
\widehat{a}_{f}=-\frac{q}{\hbar c} V, \quad \widehat{b}_{f}=\frac{i q}{\hbar c} \vec{A}
\end{gathered}
$$

for the case of a particle with charge $q$ situated within a macroscopic electromagnetic field represented by the scalar potential $V$ and vector potential $\vec{A}$. Moving all terms left-hand side (so as to obtain the pair of linear equations with null free terms), the symbolic determinant $\widehat{S}$ previously mentioned (acting upon function $\eta$ within second term of complete equation) will be

$$
\widehat{S}=\widehat{b}^{2}+\widehat{a}^{2}=\left[\frac{\partial}{\partial x}-\frac{i q \vec{A}}{\hbar c}\right]^{2}+\left[\frac{\partial}{\partial(i c t)}+\frac{q V}{\hbar c}\right]^{2} .
$$

According to Klein-Gordon equation for the relativistic wave-function of an electron in an external electromagnetic field, the result for the action of this symbolic determiant $\widehat{S}$ upon $\eta$ function should be equal to

$$
\widehat{S} \eta=\frac{(m c)^{2}}{\hbar^{2}} \eta,
$$

where $\eta$ is the associated wave function for the electron. it can be noticed that the action of the symbolic determinant $\widehat{S}$ upon this wave function equals the wave function multiplied by a constant. Thus the use of scalar and vector potentials as operators acting upon the wave functions at a later time moment within this alternating coordinate-momentum representation is justified.

As a consequence, the electromagnetic field (characterized by scalar and vector potentials $V$ and $\vec{A}$ ) should be preliminary determined in a certain area before the suggested alternating coordinate-momentum representation to be taken into consideration. No more virtual photons are necessary for computing trajectories of charged particles within an external electromagnetic field, and phase-loss phenomena (see [5]) are avoided.

\section{Conclusions}

This study has presented an alternating coordinatemomentum representation for quantum states recommended for modelling propagation and transition of associated wave functions, based on Bopp operators and on a certain symbolic determinant corresponding to a set of two linear equations with null free terms for deriving basic equations in quantum mechanics. Unlike computational aspects (see [7] where just encoding aspects are involved for a certain data set), it tries to extend input-output data correlations at possible inner dynamics of wave function. Thus a certain background dynamics could be added to the theory of light deflection based on conformal fields (see [8]). The 
long-distance interaction suggested by this dynamics based on Bopp operators is not frequency limited (as standard gaussian noise, see [9]).

It is shown that this alternating representation can justify in a good manner the patterns created through reflection/refraction of waves on nonperfectly smooth interfaces and phase correspondence of diffracted beams without the need of supplementary support functions. Correlations with Lorentz transformation of wave functions by interaction with a certain material medium (the space-time origin of a wavetrain being adjusted) are also presented, and supplementary aspects regarding the use of electromagnetic scalar and vector potentials for modelling evolution within this alternating representation are added.

Computational aspects regarding decaying modes (as presented in [10]) or a greater number of particles involved will be analysed in further studies, based on scheduling processes (as in [11]) and distributing environment techniques (as in [12]).

\section{Conflict of Interests}

The authors declare that there is no conflict of interests regarding the publication of this paper.

\section{References}

[1] E. G. Bakhoum and C. Toma, "Transient aspects of wave propagation connected with spatial coherence," Mathematical Problems in Engineering, vol. 2013, Article ID 691257, 5 pages, 2013.

[2] P. E. Sterian, "On the connection between Heisenberg's uncertainty relations and the evolution quantum equations," Buletinul Institutului Politehnic Bucureşti, vol. 46-47, pp. 43-48, 1984 (Romanian).

[3] C. Toma, "Wavelets-computational aspects of sterian-realistic approach to uncertainty principle in high energy physicsa transient approach," Internal Report, Politehnica University Bucharest, Physics Department, 2013.

[4] C. Toma, "The advantages of presenting special relativity using modern concepts," Balkan Physics Letters, vol. 5, pp. 2334-2337, 1997.

[5] G. Torres-Vega and J. H. Frederick, "Quantum mechanics in phase space: new approaches to the correspondence principle," The Journal of Chemical Physics, vol. 93, no. 12, pp. 8862-8874, 1990.

[6] G. Torres-Vega and J. H. Frederick, "A quantum mechanical representation in phase space," The Journal of Chemical Physics, vol. 98, no. 4, pp. 3103-3120, 1993.

[7] S. V. Chekanov, K. Strand, and P. Van Gemmeren, "ProMC: input-output data format for HEP applications using varint encoding," Computer Physics Communications, vol. 185, pp. 2629-2635, 2014.

[8] C. Cattani, M. Scalia, E. Laserra, I. Bochicchio, and K. K. Nandi, "Correct light deflection in Weyl conformal gravity," Physical Review D: Particles, Fields, Gravitation, and Cosmology, vol. 87, Article ID 047503, 2013.

[9] M. Li and W. Zhao, "On bandlimitedness and lag-limitedness of fractional Gaussian noise," Physica A, vol. 392, no. 9, pp. 19551961, 2013.
[10] M. Dima, "Exclusive reconstruction of semi-leptonic B-hadron decay modes," Journal of High Energy Physics, vol. 2011, article 8, 2011.

[11] V. Cristea and F. Pop, "Optimization of scheduling process in grid environments," Politehnica University Bucharest (UPB) Scientific Bulletin, Series C: Electrical Engineering, vol. 71, no. 4, pp. 35-48, 2009.

[12] C. Dobre, F. Pop, and V. Cristea, "New trends in large scale distributed systems simulation," Journal of Algorithms \& Computational Technology, vol. 5, no. 2, pp. 221-257, 2011. 


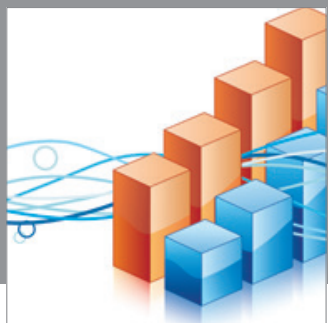

Advances in

Operations Research

mansans

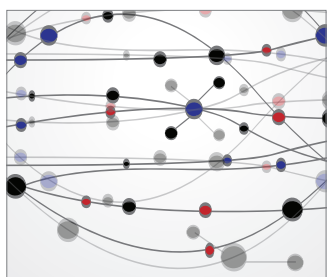

The Scientific World Journal
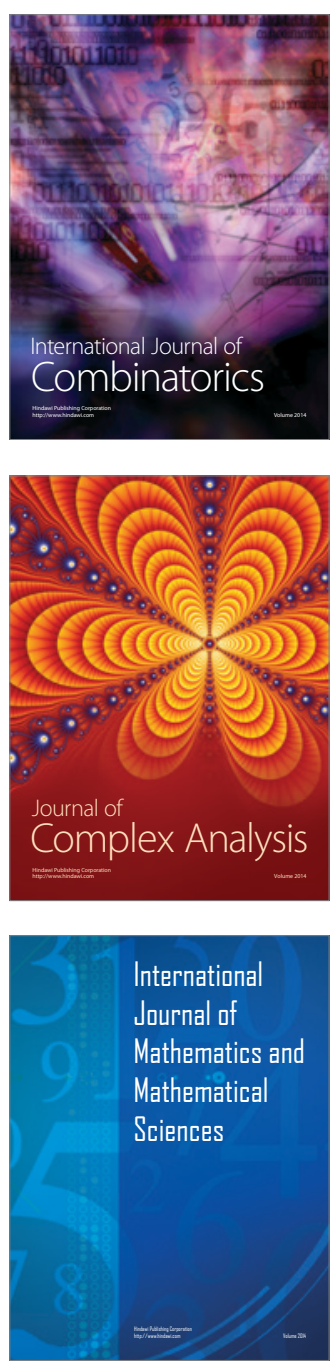
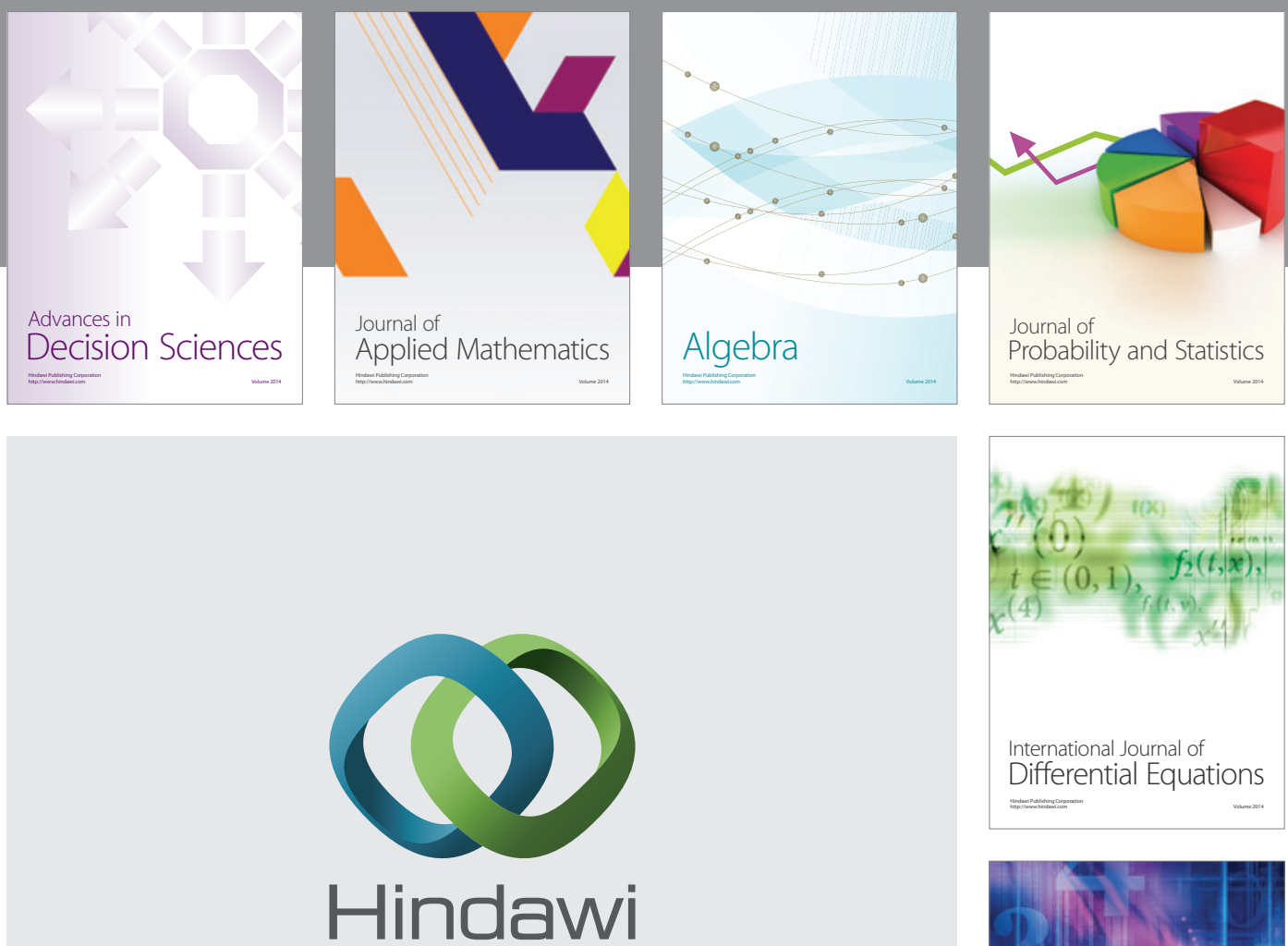

Submit your manuscripts at http://www.hindawi.com
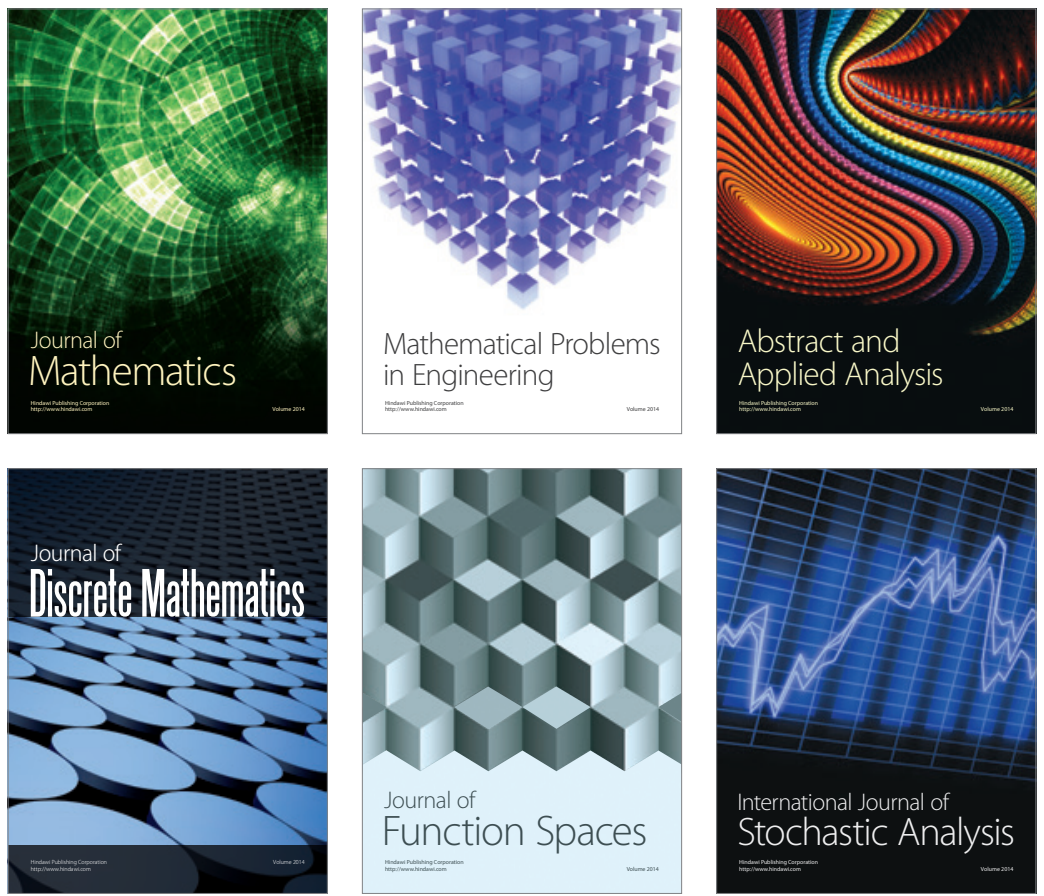

Journal of

Function Spaces

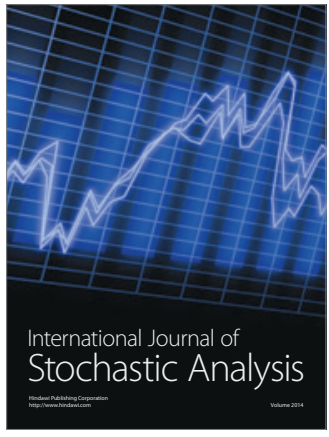

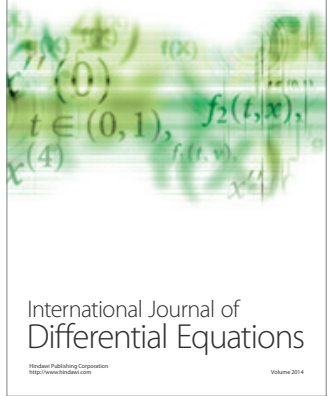
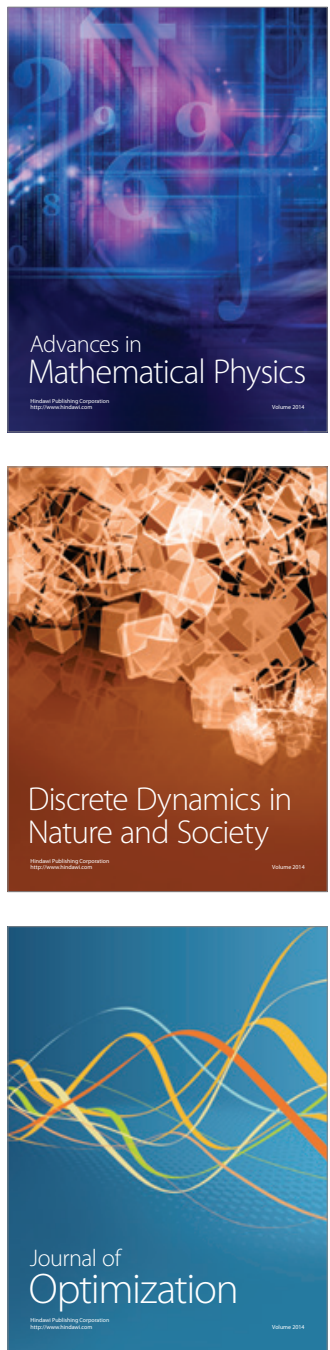